\title{
Radiofrequency catheter ablation of Mahaim tachycardia by targeting Mahaim potentials at the tricuspid annulus
}

\author{
Spencer C Heald, D Wyn Davies, David E Ward, Clifford J Garratt, Edward Rowland
}

\begin{abstract}
Background-Reentrant tachycardias associated with Mahaim pathways are rare but potentially troublesome. Various electrophysiological substrates have been postulated and catheter ablation at several sites has been described.

Objective-To assess the efficacy and feasibility of targeting discrete Mahaim potentials recorded on the tricuspid annulus for the delivery of radiofrequency energy in the treatment of Mahaim tachycardia.

Patients-21 patients out of a consecutive series of 579 patients referred to one of three tertiary centres for catheter ablation of accessory pathways causing tachycardia. All had symptoms and presented with tachycardia of left bundle branch block configuration or had this induced at electrophysiological study. In all cases, the tachycardia was antidromic with anterograde conduction over a Mahaim pathway.
\end{abstract}

Results -6 patients had additional tachycardia substrates (4 had accessory atrioventricular connections and 2 had dual atrioventricular nodal pathways and atrioventricular nodal reentry). After ablation of the additional pathways, Mahaim potentials were identified in 16 (76\%) associated with early activation of the distal right bundle branch and radiofrequency energy at this site on the tricuspid annulus abolished Mahaim conduction in all 16 cases. In 2 patients there was early ventricular activation at the annulus without a Mahaim potential but radiofrequency energy abolished preexcitation. In the remaining patients no potential could be found ( 1 patient), no tachycardia could be induced after ablation of an additional pathway (1 patient), or no Mahaim conduction was evident during the study (1 patient). During follow up (1-29 months (median 9 months)) all but 1 patient remained symptom free without medication.

Conclusions-Additional accessory pathways seem to be common in patients with Mahaim tachycardias. The identification of Mahaim potentials at the tricuspid annulus confirms that most of these pathways are in the right free wall and permits their successful ablation and the abolition of associated tachycardia.

(Br Heart f 1995;73:250-257)
Keywords: Mahaim pathways; radiofrequency ablation; tachycardia; tricuspid annulus

The advent of radiofrequency energy for catheter ablation dramatically changed the management of patients with reentrant tachycardias mediated through accessory pathways. Many now regard radiofrequency ablation as the best procedure for those with symptomatic tachycardia. Its role in Mahaim tachycardia, a rare variant of the Wolff-ParkinsonWhite syndrome, is less well defined because most Mahaim fibres exhibit electrophysiological properties that render conventional methods of mapping and ablation inapplicable.

Anomalous conduction pathways connecting the atrioventricular node or His-Purkinje system to the ventricle were first described by Mahaim over 50 years ago. ${ }^{1}$ The part played by such fibres in pre-excitation and their function in tachycardia has been the subject of considerable attention ever since ${ }^{2-5}$; they were shown to be a necessary component of the tachycardia reentrant circuit during an electrophysiological study by Gallagher et $a l^{6}$ and subsequently by others. ${ }^{78}$ When in 1984 a histological study by Gmeiner et al ${ }^{9}$ showed that these accessory tracts were present in a patient with tachycardia the link with the abnormality seemed firmly established. The clinical relevance of Gmeiner's work was thrown open to question, however, when Tchou et al ${ }^{10}$ found that the accessory pathway originated directly from the right atrium independently of the atrioventricular node in all eight patients in a surgical series. These findings have since been corroborated by others. ${ }^{11} 12$

The term Mahaim tachycardia is used to describe atrioventricular reentrant tachycardias secondary to right sided accessory pathways that show decremental anterograde conduction and participate as the anterograde limb in antidromic atrioventricular reciprocating tachycardia. Such accessory pathways may be inserted into the distal right bundle branch (atriofascicular) or directly into the ventricular myocardium near the tricuspid annulus (atrioventricular). Furthermore there is no convincing evidence of retrograde conduction in Mahaim pathways. We describe our experience in ablating the substrate for Mahaim tachycardia by targeting Mahaim potentials at the tricuspid annulus.

Patients and methods

Between December 1992 and June 1994 a total of 579 patients were referred to our 
Figure 1 Surface 12 lead electrocardiogram of Mahaim tachycardia showing typical broad complex left bundle branch block configuration.
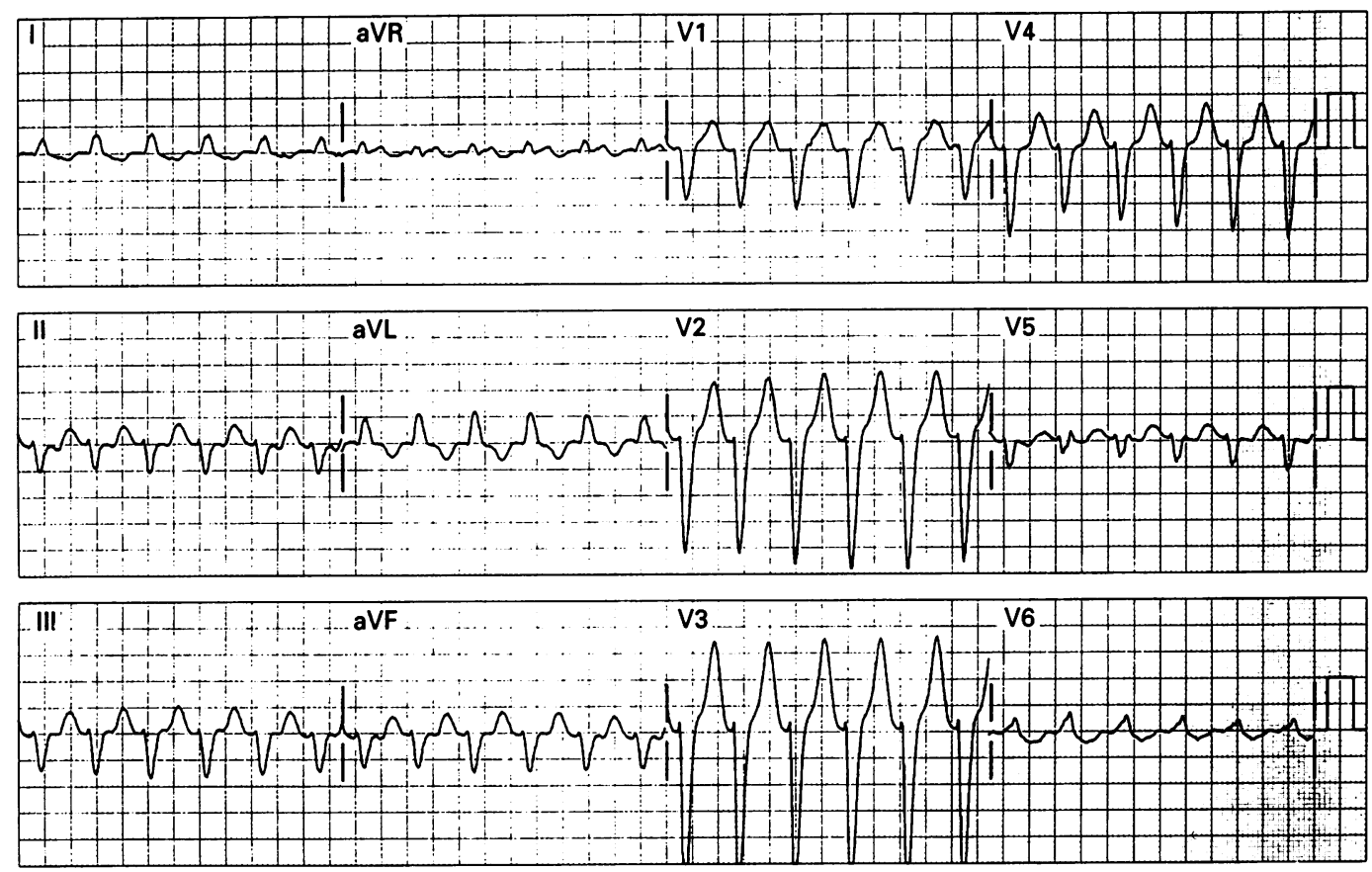

Speed: $25 \mathrm{~mm} / \mathrm{s} \quad 10 \mathrm{~mm} / \mathrm{mV}$

12 Lead ECG

hospitals to be considered for catheter ablation of an accessory atrioventricular connection. Twenty one (4\%) had Mahaim tachycardia. All had symptoms and had confirmed wide complex tachycardia (fig 1). They were aged 7-53 (median 33) and 15 were female. Twelve patients had had a previous electrophysiological study. Two of the patients had Ebstein's anomaly of the tricuspid valve; no other structural cardiac abnormality was seen. Six patients had additional tachycardia substrates: four had an additional accessory atrioventricular connection (two concealed) and two had additional slow atrioventricular nodal pathways. The resting electro-

Characteristics of the patients

\begin{tabular}{|c|c|c|c|c|c|c|c|}
\hline Case & $\begin{array}{l}\text { Age } \\
(y r)\end{array}$ & Sex & $\begin{array}{l}\text { Previous } \\
\text { EPS }\end{array}$ & $\begin{array}{l}E C G \text { in } \\
S R\end{array}$ & $\begin{array}{l}\text { Site of } \\
\text { Mahaim }\end{array}$ & $\begin{array}{l}\text { Additional } \\
\text { APs }\end{array}$ & $\begin{array}{l}\text { Tachycardia } \\
\text { mechanisms * }\end{array}$ \\
\hline 1 & 24 & $\mathbf{F}$ & Yes & $\mathbf{N}$ & Antlat $†$ & No & \\
\hline 2 & 46 & $\mathbf{F}$ & Yes & $\mathbf{N}$ & $\mathrm{NM}+$ & No & \\
\hline 3 & 18 & $\mathbf{M}$ & No & $\mathbf{N}$ & Antlat & No & \\
\hline 4 & 21 & $\mathrm{~F}$ & No & $\mathrm{N}$ & Ant & No & \\
\hline 5 & 53 & $\mathbf{M}$ & Yes & $\operatorname{Px}(M)$ & Lat & No & \\
\hline 6 & 25 & $\mathrm{~F}$ & No & $\operatorname{Px}(M)$ & Antlat & No & \\
\hline 7 & 30 & $\mathrm{~F}$ & Yes(E) & $\mathrm{N}$ & $\mathrm{NC}$ & No & \\
\hline 8 & 42 & $\mathrm{~F}$ & No(E) & $\operatorname{Px}(M)$ & Ant & No & \\
\hline 9 & 43 & $\mathrm{~F}$ & Yes & $\operatorname{Px}(M)$ & Post & RFW (conc) & OAVRT, MT \\
\hline 10 & 35 & $\mathrm{~F}$ & Yes & $\mathrm{N}$ & Ant & SP & AVNRT \\
\hline 11 & 7 & $M \ddagger$ & Yes & $\mathrm{N}$ & Antlat & SP & AVNRT, MT \\
\hline 12 & 16 & $M$ & Yes & $\mathbf{N}$ & Post & No & \\
\hline 13 & 43 & $\mathbf{M}$ & Yes & $\mathrm{N}$ & Antlat & LFW (conc) & OAVRT, MT \\
\hline 14 & 20 & F & Yes & $\operatorname{Px}(M)$ & Ant & No & \\
\hline 15 & 37 & $\mathrm{~F}$ & No & $\mathrm{Px}(\mathrm{AP})$ & Ant & PS & OAVRT, M-AP, MT \\
\hline 16 & 33 & $\mathrm{~F}$ & No & & Antlat & No & \\
\hline 17 & 23 & $M \ddagger$ & No & $\operatorname{Px}(M)$ & Ant & No & \\
\hline 18 & 33 & $\mathrm{~F}$ & Yes & $\mathrm{Px}(\mathrm{AP})$ & Antlat & PS & OAVRT, M-AP \\
\hline 19 & 14 & $\mathrm{~F}$ & Yes & $\operatorname{Px}(M)$ & Ant & No & \\
\hline 20 & 35 & $\mathrm{~F}$ & No & $\operatorname{Px}(M)$ & Post & No & \\
\hline 21 & 36 & $\mathrm{~F}$ & No & & Lat & No & \\
\hline
\end{tabular}

SR, sinus rhythm; AP, accessory pathway: (E), Ebstein's anomaly of the tricuspid valve; Px, preexcitation with letter in bracket indicating route of pre-excitation (N, normal; $M$, Mahaim); Ant, anterior; Antlat, anterolateral; Lat, lateral; Postlat, posterolateral; Post, posterior; NM, not mapped (procedural failure); NC, no evidence of Mahaim pathway conduction; PS, posteroseptal; SP, slow pathway; LFW, left free wall; RFW, right free wall; (conc), concealed accessory pathway; OAVRT, orthodromic atrioventricular reentrant tachycardia (narrow complex); MT, Mahaim tachycardia (Mahaim anterograde and AV node retrograde); AVNRT, antrioventricular nodal reentrant tachycardia (narrow complex); M-AP, Mahaim anterograde and accessory pathway retrograde.

*Tachycardia induced during ablation procedure.

tAtrioventricular Mahaim pathway.

$\ddagger$ Two ablation procedures required. cardiogram was normal in 11 patients, and showed pre-excitation over the Mahaim pathway in eight and over an additional accessory pathway in two patients. The Mahaim fibre was shown to be a direct atrioventricular connection in four (all had shown resting preexcitation over the Mahaim pathway) and an atriofascicular connection that was inserted into the distal right bundle branch in 17 (table).

\section{ELECTROPHYSIOLOGICAL STUDY AND} ABLATION PROCEDURE

After they gave informed consent all the patients underwent electrophysiological study and ablation after an overnight fast. All antiarrhythmic medication had been stopped at least five half lives before the procedure. Patients were sedated with diazepam and diamorphine.

Three or four multipolar electrodes were introduced through a femoral or subclavian vein and positioned under fluoroscopic guidance in the high right atrium, coronary sinus, right ventricular apex, and across the tricuspid valve to record the His bundle electrogram. In those who had undergone previous electrophysiological study a coronary sinus electrode was not inserted unless there was evidence of an additional left sided accessory pathway. Electograms were filtered at $30-500 \mathrm{~Hz}$ and recorded together with all 12 standard surface electrocardiographic leads on optical disc by a Bard LabSystem (C R Bard, Haverhill, Massachusetts). Programmed electrical stimulation of the right atrium and ventricle was performed with a programmable stimulator delivering rectangular pulses of $2 \mathrm{~ms}$ duration at twice diastolic threshold (Medtronic 5328, Minneapolis, Minnisota). Tachycardia induction was attempted at three basic drive cycle lengths $(600,500$, and $400 \mathrm{~ms})$ using up to two extrastimuli at progressively shorter coupling 
Figure 2 Surface electrocardiogram and intracardiac recordings during mapping in (A) a patient with an atriofascicular Mahaim pathway and (B) a second patient with a direct atrioventricular connection. (A) Intracardiac recordings from the high right atrium (HRA), distal $H$ is bundle (HBED), mid His bundle (His 2 and 3), proximal His bundle (His 4), and mapping catheter positioned at the right ventricular apex (MAPd), together with surface leads $I$, aVL, V1, and V6. During sinus rhythm (1) earliest His activation occurred proximally; in contrast, during atrial pacing with maximal preexcitation (2) the distal

$H$ is bundle leads indicated reversal of His bundle activation sequence. Early ventricular activation at the right ventricular apex during atrial pacing (3) confirmed an atriofascicular connection. (B) Intracardiac recordings from the high right atrial pacing electrode (RAP), His bundle

(HBE), mapping catheter positioned on the tricuspid annulus (MAPd), and right ventricular apex $(R V)$, together with surface ECG leads I, aVF, V1, and V6. The vertical line marks the onset of the $Q R S$ complex and ventricular activation. The His bundle potential was inscribed at the onset of local ventricular activation (1). Early ventricular activation was seen on the mapping catheter at the level of the tricuspid annulus (2), whereas apical ventricular activation was late (3), confirming a direct atrioventricular connection.

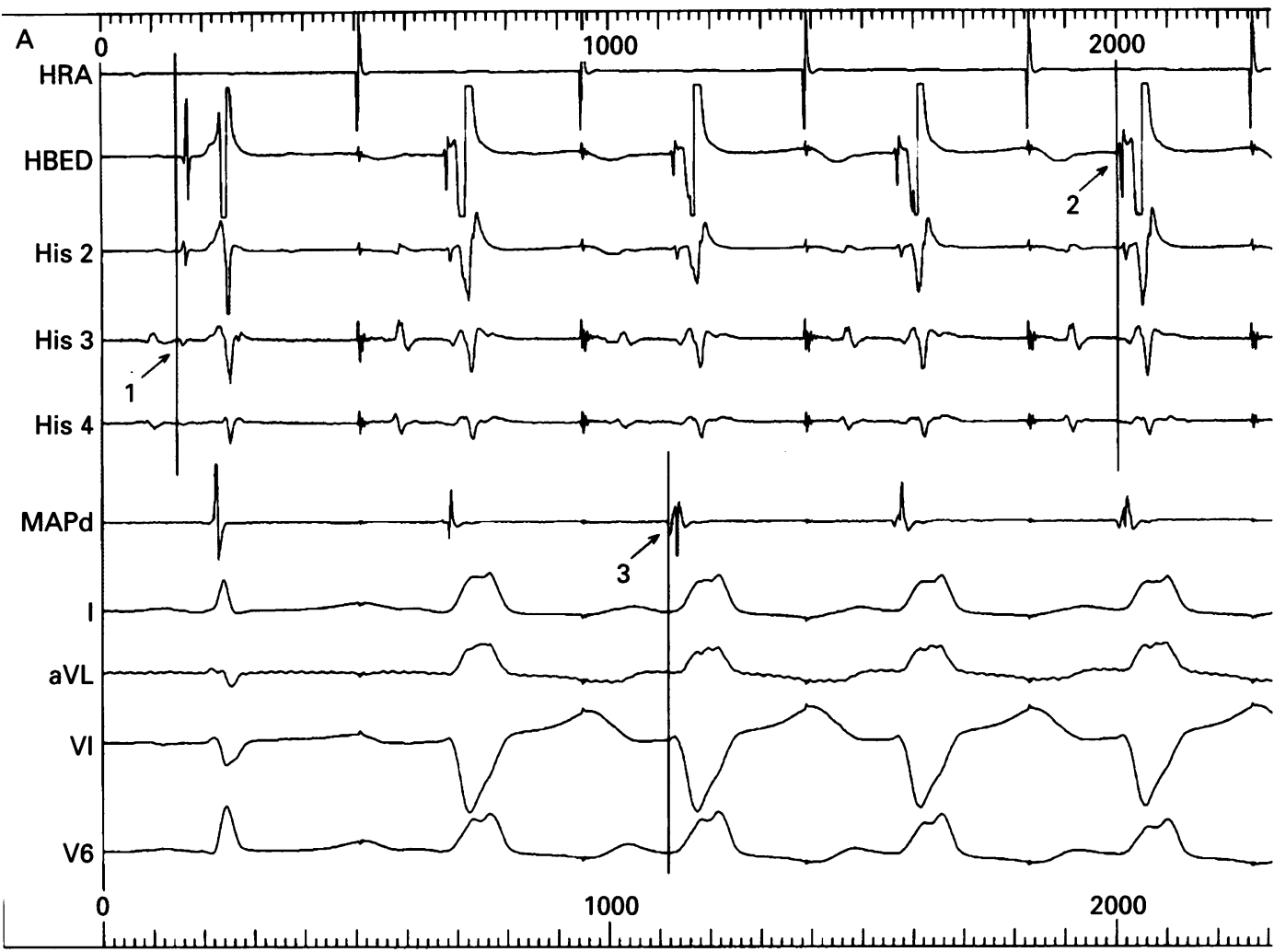

Speed: $100 \mathrm{~mm} / \mathrm{s}$

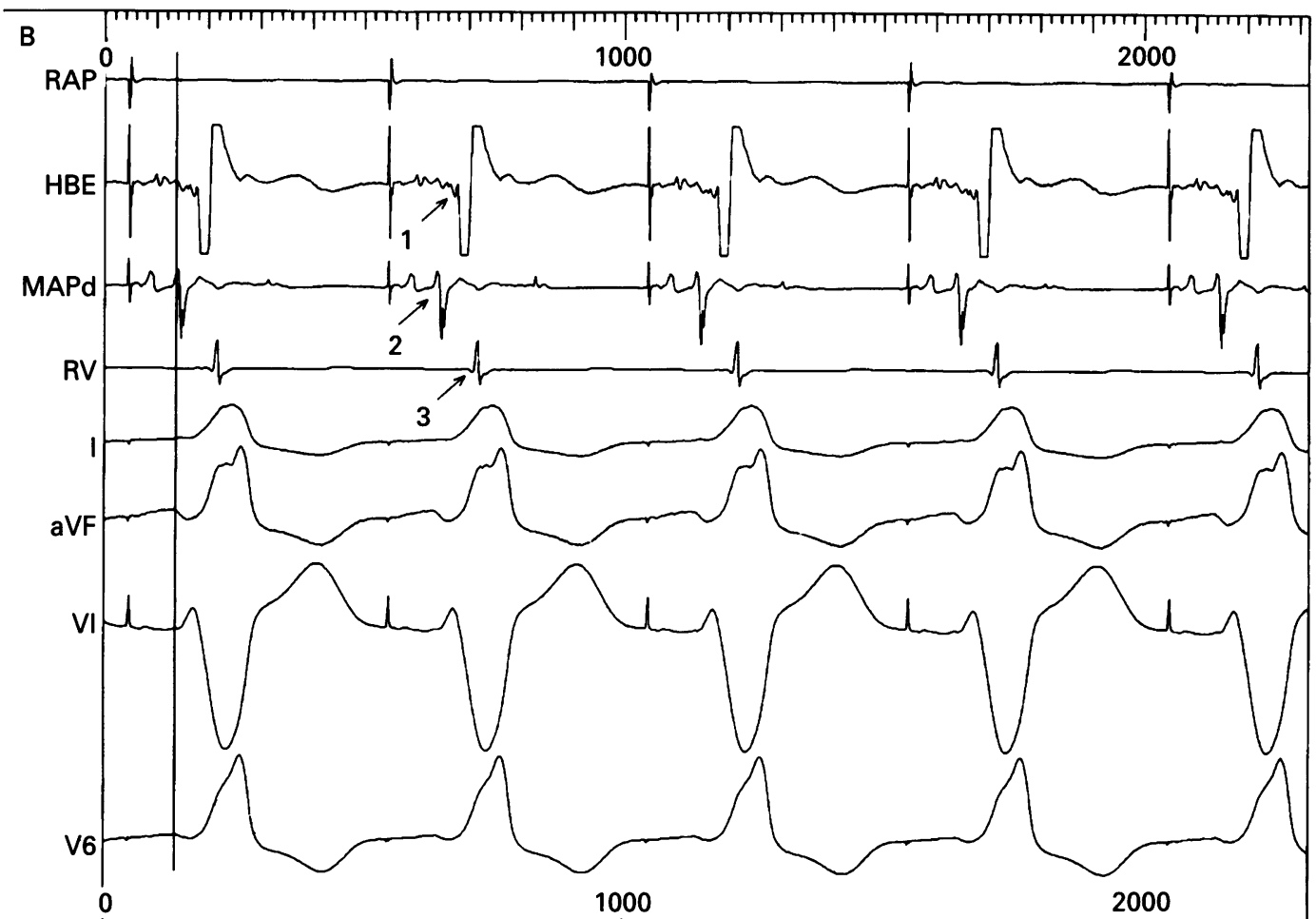

Speed: $100 \mathrm{~mm} / \mathrm{s}$

intervals. If this failed, incremental atrial or ventricular pacing was tried up to the point of atrioventricular or ventriculoatrial block.

The existence of a Mahaim pathway and of its participation as a necessary component in the reentrant circuit during tachycardia was confirmed by:

(a) A decrement in both $\mathrm{AH}$ and $\mathrm{AV}$ intervals with premature atrial stimuli, but with a lesser degree of $\mathrm{AV}$ decrement, so that the $\mathrm{HV}$ interval progressively shortened with increasing atrial prematurity.

(b) During both tachycardia and atrial pacing with maximal pre-excitation the His bundle activation sequence was seen to reverse, indicating retrograde activation of the His bundle (fig 2A).

(c) Participation as the anterograde limb in antidromic atrioventricular reciprocating tachycardia. This can be accomplished by 


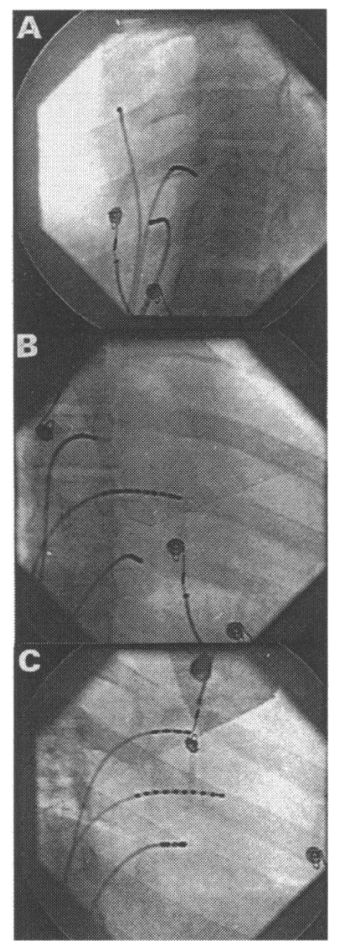

Figure 3 Cineradiograph of catheter position for ablation of a right posterior Mahaim pathway taken in the left anterior oblique (A) and right anterior oblique posteroanterior (B), and right anterior oblique $(C)$ projections. The upper catheter was positioned in the high right atrium, the middle catheter across the tricuspid valve to record His activation, and the lower catheter posteriorly on the tricuspid annulus at the site of Mahaim potential recording. Figures $4 A$ and $L B$ show corresponding electrograms.

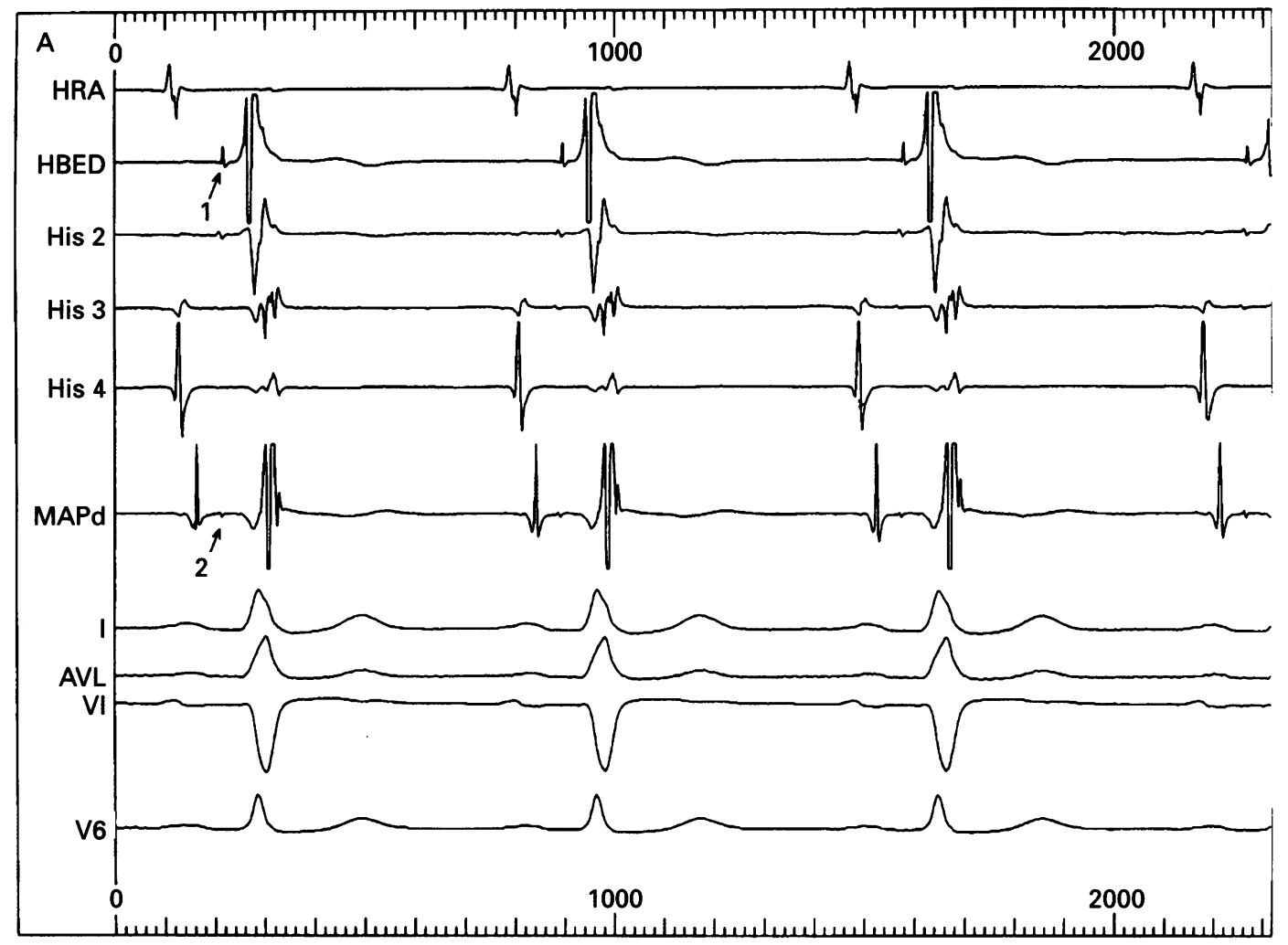

Speed: $100 \mathrm{~mm} / \mathrm{s}$

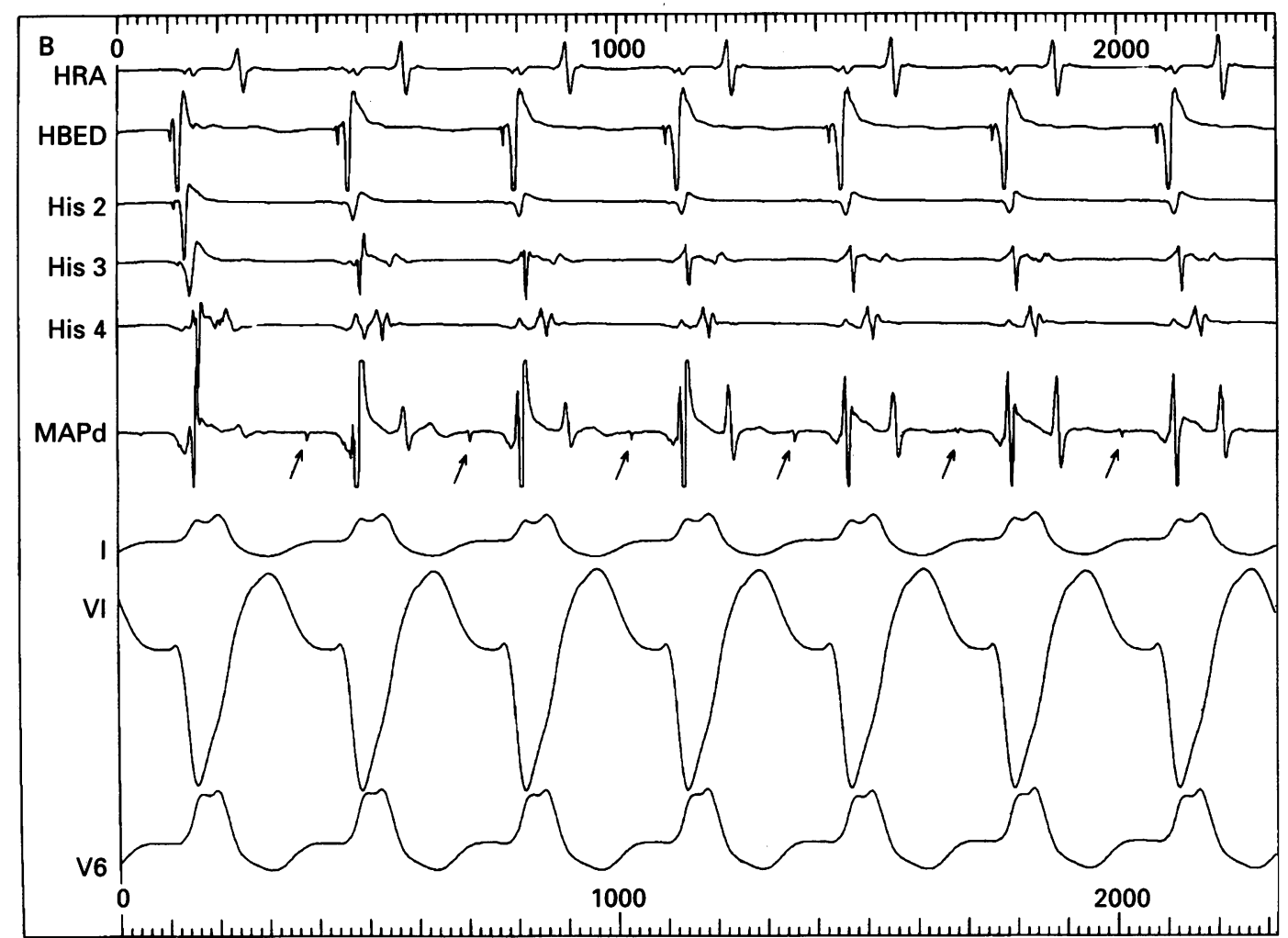

Speed: $100 \mathrm{~mm} / \mathrm{s}$

Figure 4 Intracardiac electrogram and surface electrocardiographic leads as in fig 2. (A) Sinus rhythm. A large potential was recorded from the distal His bundle region (1) and a typical discrete Mahaim pathway potential (2) from the posterior tricuspid annulus, distinct in timing from and slightly earlier than the His bundle potential. Both Mahaim pathway and anterograde His bundle activation occurred before the onset of the surface $Q R S$ complex. (B) Mahaim tachycardia. Mahaim potential (arrows) recorded during tachycardia from the same patient as in fig 4A. The Mahaim potential clearly preceded ventricular activation. The His bundle potential on the distal His bundle electrode (HBED) was clearly visible just before the local ventricular electrogram; proximal His bundle activation was obscured by the ventricular electrogram. In contrast to sinus rhythm, during tachycardia ventricular activation and surface $Q R S$ onset preceded retrograde His bundle activation. See legend to fig 2 for abbreviations. 
Figure 5 Recordings made during

radiofrequency energy

delivery. (A) Intra-cardiac

recordings from the high

right atrium and distal $H$ is

bundle, together with

surface leads I, aVL, VI

and V6. Abrupt block in

Mahaim pathway

conduction and

normalisation of the

surface $Q R S$ occurred $4 \cdot 4$

seconds after the onset of

energy delivery.

(B) Continuous recording from surface ECG lead I energy application,

showing typical pattern of stuttering block. Onset of

radiofrequency energy (1) initial block in Mahaim pathway conduction at 9.5 seconds (2); ectopic activity originating from the Mahaim pathway continued up until the end of the first 30 seconds of energy application.

Ablation at this site was successful with further energy.

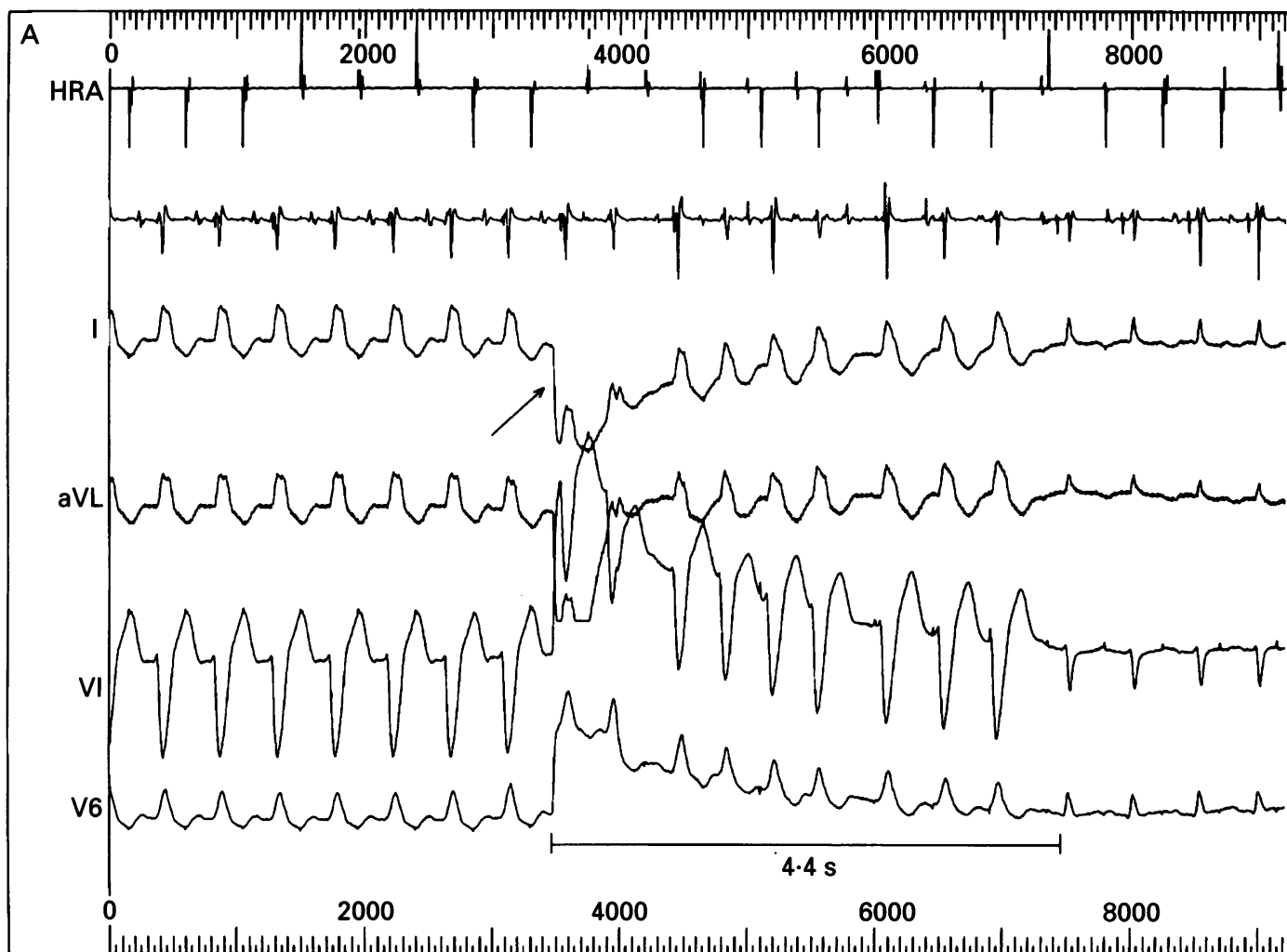

Speed: $25 \mathrm{~mm} / \mathrm{s}$

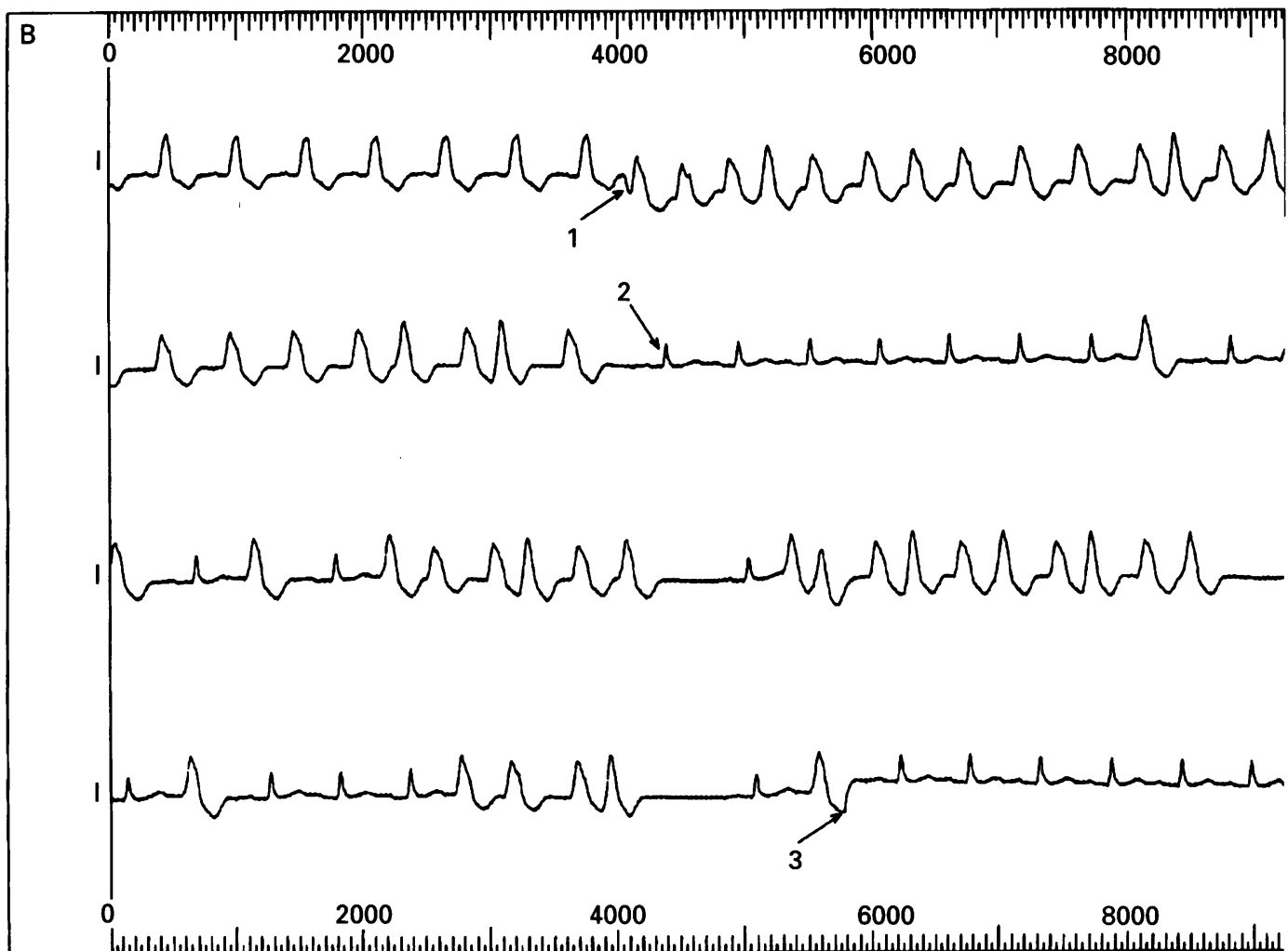

Speed: $25 \mathrm{~mm} / \mathrm{s}$

delivery of an appropriately timed atrial premature beat during tachycardia, causing advancement of ventricular activation without altering the timing of the atrial electrogram on the His bundle catheter, thus excluding atrioventricular nodal reentrant tachycardia and proving a direct atrioventricular connection.

(d) Right sided location so that pre-excited
QRS complexes and the QRS complexes in tachycardia have a left bundle branch block configuration.

During tachycardia or atrial pacing with maximum pre-excitation atriofascicular Mahaim pathways showed early ventricular activation at the right ventricular apex (fig $2 \mathrm{~A}$ ) whereas atrioventricular Mahaim connections 
showed early ventricular activation at the tricuspid annulus (fig 2B). After Mahaim tachycardia was confirmed a large-tip deflectable electrode catheter ( 7 or $8 \mathrm{~F}, 4 \mathrm{~mm}$ distal electrode, Polaris, Mansfield-Webster, Boston Scientific, Watertown, Massachusetts) was introduced through the femoral vein and passed to the right heart to enable mapping of the tricuspid annulus. Target sites for ablation were selected on the basis of the presence of discrete Mahaim potentials: these resembled signals recorded from the His bundle electrode but at sites remote from it. In addition such potentials could be temporally dissociated from the His bundle electrogram by atrial pacing and during Mahaim tachycardia. At the site of Mahaim potential recording, with the ablation catheter positioned on or slightly toward the ventricular aspect of the tricuspid annulus, we delivered a radiofrequency current $(500 \mathrm{kHz}$ unmodulated continuous sinusoidal waveform) at $20-40 \mathrm{~W}$ between the catheter tip electrode and a large indifferent electrode applied to the left posterior chest wall. Radiofrequency energy was applied for up to three and a half minutes, but delivery was stopped if no effect was seen within 15 seconds. If no Mahaim potential could be found we selected sites for ablation on the basis of stimulus-to-delta wave mapping during constant rate atrial pacing. ${ }^{13}$ If the Mahaim tachycardia was shown to be caused by a direct atrioventricular connection (earliest ventricular activation at the tricuspid annulus) we performed ablation using standard techniques for accessory pathway ablation and applied energy at the site of earliest ventricular activation. The position of the catheter tip at the successful ablation site was recorded in the $30^{\circ}$ right anterior oblique and $30^{\circ}$ left anterior oblique projections (fig 3 ).

\section{Results}

Mahaim pathway conduction was present in 20 of the 21 patients: in one in whom a previous electrophysiological study had suggested a Mahaim tachycardia, there was no evidence of accessory pathway conduction at the ablation procedure. Of the 20 patients in whom Mahaim pathway conduction was demonstrated, 16 had atriofascicular pathways and four had accessory atrioventricular connections. The site of the Mahaim pathway at the level of the tricuspid annulus was determined in 19 patients. In the remaining case early ventricular activation occurred at the tricuspid annulus, confirming an atrioventricular Mahaim pathway; but we could not determine the exact site of the pathway and the procedure failed.

Eighteen of the 21 Mahaim accessory pathways were successfully ablated and there were no significant complications. One patient is awaiting a further procedure, and in the other two tachycardia is no longer inducible, despite resting pre-excitation in one, and therefore no further intervention is planned. Mahaim potentials were identified in 16 patients during sinus rhythm or atrial pacing and in one patient they were also demonstrated during antidromic tachycardia (fig $4 \mathrm{~A}$ and $4 \mathrm{~B}$ ). In the five patients Mahaim potentials could not be identified: in two patients because there was early ventricular activation at the tricuspid annulus (that is, direct atrioventricular connection) and pre-excitation was abolished by radiofrequency energy at this site by conventional pathway mapping techniques, in one, no potential found and the procedure failed, in one, no tachycardia was inducible after ablation of an additional pathway, and in one no Mahaim conduction was evident. Procedure and fluoroscopy times (including diagnostic electrophysiological study) ranged from $49 \mathrm{~min}$ to $260 \mathrm{~min}$ (median 120), and from $7 \mathrm{~min}$ to $107 \mathrm{~min}$ (median 30) respectively. In two patients two procedures were required for successful ablation of the Mahaim pathway.

Conduction over the Mahaim pathway was abolished either abruptly or more typically, in a stuttering fashion with ectopic activity that had a QRS morphology identical to the Mahaim pre-excitation pattern (fig 5A and B). A stuttering block was seen in 12 cases. Radiofrequency energy was applied at 1-13 sites (median 3 ) and for up to 210 s (median 90) at the successful site. After abolition of conduction down the Mahaim pathway we waited $30 \mathrm{~min}$ before repeating the electrophysiological testing to confirm successful ablation.

In five patients pre-excitation over the Mahaim pathway was abolished by the pressure of the catheter tip. In these patients radiofrequency energy was applied at the site of mechanical block and the waiting period was extended to up to 2 hours. Despite energy delivery at this site conduction over the Mahaim pathway recurred within 2 hours in three patients: in these patients electrogram guided ablation was successfully performed during the same procedure. In two patients a traumatic block persisted for $>24$ hours: one patient presented 3 weeks later with recurrent tachycardia and ablation was successful 3 months after the initial procedure and in the other pre-excitation had recurred by the 6 week follow up visit. In this patient tachycardia was not inducible at a repeat electrophysiological study so no further procedure is planned. During a follow up of from 1 to 29 months (median 9 months) there has been no other late recurrence of conduction in either the Mahaim pathway or other ablated tachycardia substrates.

Four patients showed evidence of an additional accessory atrioventricular connection; in all this connection was shown to be responsible for the dominant tachycardia at the start of the procedure and these additional pathways were ablated before ablation of the Mahaim fibre was attempted. Two patients had anterogradely conducting posteroseptal accessory pathways; in both these were responsible for the pattern of pre-excitation in sinus rhythm and they were ablated by standard techniques. Similarly, in the patients with a concealed left and right free wall 
accessory pathway, mapping and ablation were performed in the usual manner during ventricular pacing. In one of the patients with an additional slow atrioventricular nodal pathway the slow pathway was successfully ablated before the Mahaim fibre. In the other patient with additional atrioventricular nodal reentrant tachycardia pre-excitation via the Mahaim fibre could not be demonstrated during sinus rhythm or with atrial pacing. The dominant arrhythmia was typical atrioventricular nodal reentrant tachycardia although non-sustained Mahaim tachycardia was seen on three occasions, during which retrograde conduction was via the fast atrioventricular nodal pathway. Therefore, because in the absence of pre-excitation the Mahaim pathway could not be mapped, we decided to ablate the common retrograde limb of both tachycardias - the fast atrioventricular nodal pathway. This resulted in a ventriculoatrial block so that neither tachycardia was inducible and it unmasked the pre-excitation caused by the Mahaim pathway.

\section{Discussion}

This consecutive series of 21 patients showed that radiofrequency ablation is a highly successful procedure for the treatment of a rare but potentially lethal arrhythmia. ${ }^{9}$ Consistent with other series describing catheter-based or surgical techniques for the treatment of Mahaim tachycardias none of our subjects was found to have the substrate as originally described by Mahaim. ${ }^{1}$ In fact, it may be that the atrioventricular fibres originally described by Kent are the substrate for Mahaim tachycardia, these accessory bundles had the histological characteristics of atrioventricular nodal tissue. ${ }^{14}$ Nevertheless the term "Mahaim tachycardia", as applied to the syndrome of a right sided accessory pathway with decremental anterograde conduction and participation of this pathway as the anterograde limb in a wide QRS complex tachycardia of left bundle branch block configuration, has become so entrenched that it would be impractical to rename it.

Haissaguerre et al reported successful catheter ablation of Mahaim fibres in three patients by high energy direct current shocks targeted on the distal insertion of the atriofascicular pathway. ${ }^{15}$ Two patients in this small series developed permanent right bundle branch block. Furthermore with this approach the distal right bundle branch may be ablated without destroying the atriofascicular fibre. This may result in a tachycardia with a longer cycle length (retrograde conduction via the left bundle branch) and possibly incessant tachycardia.

Klein et al described the successful ablation of three atriofascicular and one atrioventricular Mahaim fibres when radiofrequency energy was delivered at the tricuspid annulus. ${ }^{13}$ The ablation site on the tricuspid annulus was selected during either constant rate atrial pacing, in which the operator searches for the site at which a premature atrial beat produces the shortest stimulus-to-delta interval, or alternatively during tachycardia by locating the site at which the latest premature atrial beat could advance ventricular activation without affecting the timing of the His bundle atrial electrogram. Both of these mapping techniques are complicated and inherently imprecise; as is borne out by the large number of radiofrequency energy deliveries required to achieve success in Klein's series (range 10-19).

In most of our patients, except for the cases with a direct atrioventricular connection, the target site for ablation was identified on the basis of what was presumed to be a discrete Mahaim potential. Mahaim potentials have been described elsewhere, ${ }^{1617}$ and ablation of a Mahaim fibre after localisation of Mahaim potentials has been described in a case report, ${ }^{18}$ but to our knowledge this is the first large prospective study of the feasibility of ablating a Mahaim pathway by targeting such potentials for radiofrequency energy delivery. We have never encountered such potentials on mapping of the tricuspid annulus during ablation procedures for typical non-decremental accessory atrioventricular connections, and furthermore Mahaim pathway ablation was successful in all patients in whom Mahaim potentials could be recorded. We believe that this method is the best way to ablate Mahaim tachycardias because it is likely to require fewer energy deliveries, because it uses simpler mapping techniques, and because it avoids the potential complications of ablating in the region of the distal right bundle branch.

Nearly a third of our patients had several arrhythmia substrates. This accords with other reports that those with Mahaim tachycardia often have additional pathways. ${ }^{19-21}$ This raises the problem of which pathway to ablate first. In general culprit pathways should be considered the primary target for ablation. Those in whom additional accessory pathways are responsible for the dominant pattern of pre-excitation can have mapping and ablation in sinus rhythm. Ablation to remove the additional arrhythmia is justified in those with concealed accessory pathways or atrioventricular nodal reentry when the additional pathway is shown to support tachycardia. This logical sequential approach to ablation led to one Mahaim pathway not being ablated because tachycardia could no longer be initiated after modification of the fast pathway of the atrioventricular node.

Pressure exerted by the catheter tip of the mapping electrode can abolish conduction in both normal and accessory pathways. Transient right bundle branch block is not uncommon during electrophysiological studies: however, in our experience traumatic block is much more common in Mahaim pathways than in typical accessory atrioventricular connections. Okishige et al reported successful ablation of an atriofascicular fibre by application of radiofrequency energy on the ventricular side of the tricuspid annulus at a site where catheter tip pressure caused fortuitous disappearance of pre-excitation. $^{22}$ 
More recently it has even been suggested that for Mahaim pathways catheter-induced mechanical block is the best marker to guide radiofrequency energy delivery at the tricuspid annulus. ${ }^{23}$ For a number of reasons we cannot recommend this approach. Firstly, in two cases in our series traumatic block persisted for $>24$ hours and possibly even as long as several weeks and in another case it persisted for nearly 2 hours. Secondly, because of the inherent catheter instability in mapping right sided accessory pathways at the tricuspid annulus, there is no guarantee that the catheter remains positioned at the critical site after the traumatic block develops-it may merely have brushed across the site. Thus there is no certainty that radiofrequency energy application delivered after mechanical block will destroy the pathway. In our series radiofrequency energy delivered after traumatic block did not result in long-term success, and we therefore recommend waiting for up to 2 hours until conduction down the Mahaim pathway resumes and mapping may proceed or the procedure is abandoned.

Stuttering block in conduction down the Mahaim pathway during radiofrequency energy delivery was seen in 12 cases in this series, accompanied by frequent runs of Mahaim extrasystoles. Such runs of ectopic rhythm no doubt increase catheter instability on the tricuspid annulus and may necessitate prolonged energy delivery, however, they also seem to be a hallmark of success because conduction did not recur in these patients. Perhaps runs of Mahaim ectopic rhythm during radiofrequency application should be regarded in much the same way as junctional rhythm accompanying slow pathway ablation for atrioventricular nodal reentry.

\section{Conclusions}

It is effective and safe to use radiofrequency energy to ablate Mahaim tachycardia (a rare but potentially troublesome arrhythmia) by targeting Mahaim potentials at the level of the tricuspid annulus. The targeting of Mahaim potentials provides a simpler method of approach than that reported in earlier series: it can reduce procedure and fluoroscopy times. Additional arrhythmia substrates are common and necessitate a stepwise logical approach targeting the culprit arrhythmia. Mechanically induced block is more common in the Mahaim pathway than in typical accessory atrioventricular pathways, and stuttering block in Mahaim pathway conduction during application of radiofrequency energy is frequent.
1 Mahaim I, Winston MR. Recherches d'anatomie comparée et de pathologie experimentale sur les connexions hautes du faisçeau de His-Tarawa. Cardiologia 1941;5:189-260.

2 Tonkin AM, Dugan FA, Svenson RH, Sealy WC, Wallace AG, Gallagher JJ. Coexistence of functional Kent and Mahaim-type tracts in the pre-excitation syndrome: demonstration by catheter techniques and epicardial demonstration by catheter techniques

3 Lev M, Fox SM, Bharati S, Greenfield JC, Rosen KM, Pick A. Mahaim and James fibers as a basis for a unique variety of ventricular preexcitation. $A m \quad \mathcal{f}$ Cardiol 1975;36:880-8.

4 Touboul P, Vexler RM, Chatelain MT. Re-entry via Mahaim fibres as a possible basis for tachycardia. $\mathrm{Br}$ Heart $\mathcal{f} 1978 ; 40: 806-11$.

5 Ward DE, Camm AJ, Spurrell RAJ. Ventricular preexcitation due to anomalous nodoventricular pathways: repor of three patients. Eur $\mathcal{F}$ Cardiol 1979;9:111-27.

6 Gallagher JJ, Smith WM, Kasell JH, Benson DW, Sterba R, Grant AO. Role of Mahaim fibers in cardiac arrhythmias in man. Circulation 1981;64:176-89.

7 Gillette PC, Garson A, Cooley DA, McNamara DG Prolonged and decremental antegrade conduction properties in right anterior accessory connections: wide QRS erties in right anterior accessory connections: wide QRS
antidromic tachycardia of left bundle branch block patantidromic tachycardia of left bundle branch block pattern without Wolff-Parkinson-White con
sinus rhythm. Am Heart $\mathcal{1}$ 1982;103:66-74

8 Bardy GH, German LD, Packer DL, Coltorti F, Gallagher JJ. Mechanism of tachycardia using a nodofascicula Mahaim fiber. Am f Cardiol 1984;54:1 140-1.

9 Gmeiner $\mathrm{R}$, Choi Keung $\mathrm{Ng}$, Hammer I, Becker AE Tachycardia caused by an accessory nodoventricula tract: a clinico-pathological correlation. Eur Heart $f$ 1984;5:233-42.

10 Tchou P, Lehmann MH, Jazayeri $M$, Akhtar $M$. Atriofascicular connection or a nodoventricular Mahaim fiber? Electrophysiologic elucidation of the pathway and associated reentrant circuit. Circulation 1988;77:837-48.

11 Klein GJ, Guiraudon GM, Kerr CR, et al. "Nodoventricular" accessory pathway: evidence for a distinct accessory atrioventricular pathway with atridistinct accessory atrioventricular pathway with atri-
oventricular node-like properties. $尹 \mathrm{Am}$ Coll Cardiol 1988;11:1035-40.

12 Ross DL, Denniss AR, Johnson DC, Sadick N, Richards DA, Uther JB. Further observations on Nodo-ventricular fibres: anatomic location and electrophysiology [abstr]. Circulation 1989;80 (suppl II):432 .

13 Klein LS, Hackett FK, Zipes DP, Miles WM. Radiofrequency catheter ablation of Mahaim fibres at the tricuspid annulus. Circulation 1993;87:738-47.

14 Kent AFS. Researches on the structure and function of the mammalian heart. $\mathcal{F}$ Physiol 1893;14:232--54.

15 Haissaguerre $\mathrm{M}$, Warin JF, Le Metayer $\mathrm{P}$, et al. Catheter ablation of Mahaim fibers with preservation of atrioventricular nodal conduction Circulation 1990;82:418-27.

16 Tchou P, Keim S, Kinn RM, Rist KE, Setter SF, Adhar GC. Electrophysiologic evidence for an ectopic nodeHis like atrioventricular conduction system in an atrioHascicular pathway [abstr]. PACE 1992;15:517.

17 McClelland JH, Jackman WM, Beckman KJ, et al. Direct recordings of right atriofascicular accessory pathway (Mahaim) potentials at the tricuspid annulus [abstr] PACE 1992;15:548.

18 Mounsey JP, Griffith MJ, McComb JM. Ablation of a Mahaim fibre following localisation of Mahaim pathway potentials. $\mathcal{F}$ Cardiovasc Electrophysiol 1994;5:432-7.

19 Sung RJ, Stypereck JL. Electrophysiologic identification of dual atrioventricular nodal pathway conduction in patients with reciprocating tachycardia using anomalous bypass tracts. Circulation 1979;60:1464-76.

20 Lerman BB, Waxman HL, Proclemer A, Josephson ME Supraventricular tachycardia associated with nodoventricular and concealed atrioventricular bypass tracts. $\mathrm{Am}$ tricular and concealed atriovent

21 Murabit I, Sosa E, Pileggi F, Denes P. Multiple reentry tachycardia in patients with ventricular-preexcitation: Report of three cases. Am Heart $₹$ 1986;111:69-80.

22 Okishige K, Strickberger SA, Walsh EP, Saul JP, Friedman PL. Catheter ablation of the atrial origin of a decrementally conducting atriofascicular accessory pathway by radiofrequency current. $\mathcal{f}$ Cardiovasc Electrophysiol 1991;2:465-75.

23 Cappato R, Schluter M, Weiss C, Kuck K. Radiofrequency current ablation at the tricuspid annulus of so-called Mahaim fibers guided by catheter-induced mechanical conduction block [abstr]. $\mathcal{F}$ Am Coll Cardiol 1994;23:277A. 